\title{
Top physics at Future Circular Colliders
}

\author{
Clement Helsens* \\ CERN, CH-1211 Geneva 23, Switzerland \\ E-mail: clement.helsens@cern.ch
}

The physics reach and feasibility of the Future Circular Colliders (FCC) are currently being investigated in the form of Conceptual Design Reports. The ultimate goal of FCC is to collide protons with a centre-of-mass energies of $100 \mathrm{TeV}$, thus extending the reach of the current HEP facilities. This high-energy regime opens new opportunities for the discovery of physics beyond the Standard Model (SM), but also for precise measurement of fundamental parameters of the SM. Following the LEP/LHC model, it is obvious that the first collider that would be operated, is an electron positron machine, FCC-ee. It aims at collecting multi-ab ${ }^{-1}$ integrated luminosities in $e^{+} e^{-}$collisions at $\sqrt{s}=90,160,240$, and $350 \mathrm{GeV}$, yielding $10^{12} \mathrm{Z}$ bosons, $10^{8} \mathrm{~W}^{+} \mathrm{W}^{-}$pairs, $10^{6}$ Higgs bosons and $10^{6} t \bar{t}$ pairs. This document presents a glimpse of what the top physics program could be at Future Circular Colliders.

The 39th International Conference on High Energy Physics (ICHEP2018)

4-11 July, 2018

Seoul, Korea

${ }^{*}$ Speaker. 


\section{Introduction}

The Futur Circular Colliders (FCC) project [1] is a 100 kilometre tunnel in the Geneva area. It is currently being investigated in the form of a Conceptual Design Report.[2]

FCC-ee will collide $e^{+} e^{-}$and operate at centre-of-mass energy ranging from $\sqrt{s}=90$ to $365 \mathrm{GeV}$. With the multi-ab ${ }^{-1}$ integrated luminosities collected at the different centre-of-mass energy points, it will provide a 20 to 50 fold improvements in many SM parameters, allows highprecision model-independent determination of the Higgs couplings, total width, and exotic and invisible decays [3]. Even if not produced directly, it will be possible to access Higgs self-coupling through loop corrections in HZ production [4]. First-generation fermion couplings (u,d,s) through exclusive decays $\mathrm{H} \rightarrow V \gamma,(V=\rho, \omega, \phi)[5]$ are also accessible.

FCC-hh would mainly collide protons with a centre-of-mass energy $\sqrt{s}=100 \mathrm{TeV}$. To achieve this goal massive research and development is needed to accomplish a robust design of 16 Tesla dipole magnets. With this unprecedented energy it will be possible for example to search for heavy resonances up to masses of the order of $40 \mathrm{TeV}$ and super-symmetric top partner up to $m \sim 10 \mathrm{TeV}$ [6]. It will also be possible to measure with high precision the Higgs self-coupling, and Higgs rare decays as well as electro-weak and top physics in extreme dynamical regimes [7, 8].

\section{Top physics at FCC-ee}

Top quarks have never been produced at lepton colliders, thus with sufficient energy a completely new set of measurements opens up. The running conditions would be a dedicated run of $\sim 1.5 \mathrm{ab}^{-1}$ at and around the $t \bar{t}$ threshold of $350 \mathrm{GeV}$. This would be complemented by a specific run of $\sim 0.2 \mathrm{ab}^{-1}$ for measurements at threshold scan and possibly run at $365 \mathrm{GeV}$ for top coupling measurement $(t \bar{t} Z, t \bar{t} \gamma, t \bar{t} H)$. The $t \bar{t}$ cross-section at threshold is approximately $0.55 \mathrm{pb}$, and considering $1.7 \mathrm{ab}^{-1}$ (6 years of running), would translate in $\sim 10^{6}$ high purity top-pair events. Top physics program will include precise measurements that coupled with precise theoretical calculation provides excellent discovery potential and at the same time is a portal to new physics effects at high scales. The extremely clean environment and large statistics at FCC-ee will allow to probe for example, anomalous couplings, indirect effects from loop contributions, suppressed and rare decays (from very clean final states).

The $t \bar{t}$ cross-section at threshold is highly sensitive to the top mass, top width, $\alpha_{s}$ and topYukawa and can thus be calculated with high precision. The measurement of the top-pair production cross-section will be done at different energy points in the threshold region. The default assumption is that each energy point will get equal integrated luminosity, but it is important to point out that the optimal way to distribute the integrated luminosity depends on the variables. The derivative of the cross-section is shown on Figure 1 left for various parameters and is normalised to typical changes of these parameters. For the measurements, the uncertainties from theory that are considered, arises from scale variations leading to $\sim 45 \mathrm{MeV}$ systematic on the top-quark mass. The scale uncertainties are roughly equivalent to a $3 \%$ luminosity uncertainty, which needs to be known on the few per-mille. The beam energy leads to an effective shift of the curve which directly translates to mass value. The goal is to keep it below $10 \mathrm{MeV}$. In summary, with $\sim 0.2 \mathrm{ab}^{-1}$, 
FCC-ee can achieve a $45 \mathrm{MeV}$ uncertainty on the top-quark mass and a $17 \mathrm{MeV}$ uncertainty on the top-quark width.

Top electro-weak couplings, $t \bar{t} Z, t \bar{t} \gamma$, are enhanced in extra dimensions, composite Higgs models and can be directly probed in production at FCC-ee. The large statistics and final state polarisation allow a full separation of the $t \bar{t} Z / \gamma$ couplings with no need for polarisation in the initial state. It has been found that the optimal centre-of-mass energy is $365 \mathrm{GeV}$ [9]. The measurement includes conservative assumptions on the detector performance. Theory uncertainty on production mechanism dominates and the precision at which FCC-ee is expected to measure it is of order $10^{-2}$ to $10^{-3}$.

The prospective in terms of electro-weak fits after FCC-ee will improve the understanding and consistency of the SM in top-W-H radiative corrections and will provide sensitivity to new physics scales up to $70 \mathrm{TeV}$.
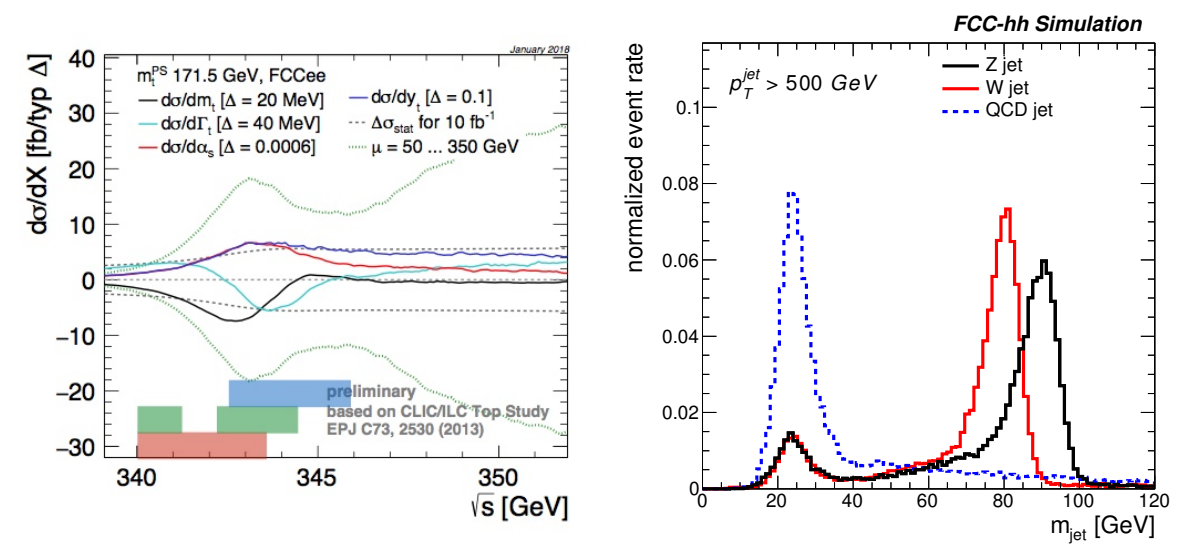

Figure 1: Left: Derivative of the $t \bar{t}$ cross-section as a function of $\sqrt{s}$ normalised to typical changes of these parameters. The coloured regions shows the area sensitive to mass (red) width (green) and Yukawa (blue). Right: Distribution of the jet mass for boosted $\mathrm{W}, \mathrm{Z}$ and QCD jet with $p_{T}>500 \mathrm{GeV}$ without magnetic field.

\section{Top physics at FCC-hh}

At $\sqrt{s}=100 \mathrm{TeV}$ pp collisions, the top pair cross-section is dominated by gluon-gluon fusion and is 45 times larger than at LHC. Considering an integrated luminosity of $20 \mathrm{ab}^{-1}$, we would get $\sim 10^{13}$ top-pairs, so $\sim 10^{13} \mathrm{~W}$-boson and b-jets. W-bosons decaying to tau leptons could be used to study rare decays and CPV for example. At high invariant mass of the $t \bar{t}$ system, $\left(m_{t \bar{t}}>15 \mathrm{TeV}\right), q \bar{q}$ production mode dominates and around $2 \cdot 10^{4}$ events will be produced with $20 \mathrm{ab}^{-1}$, which are extremely interesting for possible new physics at high $m_{t \bar{t}}$. At $100 \mathrm{TeV}$ topquarks tend to be produced at larger rapidity than at $14 \mathrm{TeV}$. This suggests that the top quarks will be a copious source of large rapidity lepton, and it is important to make sure detectors cover well these regions. A boosted two body decay from massive object has a typical angular size $\Delta R \sim 2 m / p_{T}$. For the top-quark it translates at the LHC for a typical $p_{T}$ of $1 \mathrm{TeV}$ to $\Delta R=0.5$. At FCC, the typical $p_{T}$ is ten times larger, thus $\Delta R=0.05$. For W/Z bosons with the same $p_{T}, \Delta R$ is two times smaller. This calls for a factor of 10 in granularity for the detectors. Top-quark carries 
colour charge and undergoes final state radiation. Thus soft contamination from underlying events, initial state radiation and pile-up can produce large corrections to the top mass and it is necessary to apply grooming techniques (pruning, soft drop, trimming). Figure 1 shows the jet mass for high $p_{T}$ objects in the a full simulation of the calorimeter and without magnetic field. It shows that with simplistic analysis some discrimination between $\mathrm{W}$ and $\mathrm{Z}$ boson can be observed and that very good separation with prompt QCD jets is achieved. The next steps would be to explore more sophisticated methods including particle-flow and machine learning as tagging boosted object at FCC-hh is one of the key requirement.

\section{Summary}

At FCC-ee, top threshold scan is one of the core measurement, as it enables the precise exploration of top quark properties, with small theoretical uncertainties. A precision of $10 \mathrm{MeV}$ on the top-mass can be achieved, and few percent on the $t \bar{t} Z, t \bar{t} \gamma$ electro-weak couplings. Given the large and clean dataset, strong limits on flavour changing neutral current of $10^{-4} / 10^{-5}$ can be reached for $t q \gamma$ and $t q Z$ respectively.

At FCC-hh tens of trillions of top-quarks will be produced, and such high statistics can be used to target unexplored corners of the phase space. On the contrary to common belief, high energy proton colliders are suitable for precision physics.

Top processes at Future Circular Colliders have excellent sensitivity to physics beyond the Standard Model. A precise characterisation of these processes may deliver the unexpected discovery that this field needs. Energy-frontier $e^{+} e^{-}$and p p machines both offer exciting new possibilities, with excellent sensitivity to high-scale new physics.

\section{References}

[1] M. Benedikt, Study status and further plans, talk at FCC week https://indico.cern.ch/event/656491/timetable/, Amsterdam, 2018

[2] FCC Design Reports - Contributor Registration https://indico.cern.ch/event/750953/overview

[3] M. Bicer et al., First Look at the Physics Case of TLEP, JHEP 1401, 164 (2014) [arXiv:1308.6176 [hep-ex]].

[4] M. McCullough, An Indirect Model-Dependent Probe of the Higgs Self-Coupling, Phys. Rev. D 92 (2015) no.3, 039903 [arXiv:1312.3322 [hep-ph]].

[5] A. L. Kagan, G. Perez, F. Petriello, Y. Soreq, S. Stoynev and J. Zupan, Exclusive Window onto Higgs Yukawa Couplings, Phys. Rev. Lett. 114 (2015) no.10, 101802 [arXiv:1406.1722 [hep-ph]].

[6] T. Golling et al., Physics at a $100 \mathrm{TeV}$ pp collider: beyond the Standard Model phenomena, CERN Yellow Report (2017) no.3, 441 [arXiv:1606.00947 [hep-ph]].

[7] R. Contino et al., Physics at a $100 \mathrm{TeV}$ pp collider: Higgs and EW symmetry breaking studies, CERN Yellow Report (2017) no.3, 255 [arXiv:1606.09408 [hep-ph]].

[8] M. L. Mangano et al., Physics at a $100 \mathrm{TeV}$ pp Collider: Standard Model Processes, CERN Yellow Report (2017) no.3, 1 [arXiv:1607.01831 [hep-ph]].

[9] P. Janot, Top-quark electroweak couplings at the FCC-ee, JHEP 1504 (2015) 182 [arXiv:1503.01325 [hep-ph]]. 